\title{
The relationship of serum 25 -hydroxyvitamin $D$ with glucose homeostasis in obese children and adolescents in Zhejiang, China
}

\author{
Ke Huang ${ }^{1 *}$, You-Jun Jiang ${ }^{1}$, Jun-Fen Fu' ${ }^{1}$ Jian-Feng Liang ${ }^{2}$, Hong Zhu ${ }^{3}$, Li-Fei Hu ${ }^{3}$, Zhi-Wei Zhu ${ }^{3}$ Guan-Pin Dong ${ }^{1}$, \\ Xue-Feng Chen ${ }^{1}$
}

From 8th APPES Biennial Scientific Meeting

Darwin, Australia. 29 October - 1 November 2014

\begin{abstract}
Aims
Evidence of the association between vitamin D, insulin resistance and oral disposition index (oDI) in obese children and adolescents is limited. We investigated serum $25(\mathrm{OH}) \mathrm{D}$ levels in obese children and adolescents in Zhejiang, China, and determined the relationship between serum $25(\mathrm{OH}) \mathrm{D}$ and glucose metabolism.
\end{abstract}

\section{Method}

A cross-sectional design was used. All together 348 obese and 445 non-obese children and adolescents (aged from 6-16 years old) were enrolled in this study. Obese children were divided into four subgroups: normal glucose tolerance (NGT), isolated impaired fasting glucose (IFG), isolated impaired glucose tolerance (IGT), combined IFG and ITG (IFG+ITG) according to the oral glucose tolerance test. We measured serum $25(\mathrm{OH}) \mathrm{D}$ levels and calculated the homeostasis model of insulin resistance (HOMA-IR), the whole body insulin sensitivity index (WBISI), the product of $\beta$-cell function and insulin sensitivity by the disposition index (DI).

\section{Results}

The levels of 25(OH)D in obese group were significantly lower than those of non-obese group; serum 25(OH)D level in obese with NGT group was higher than that of the other three subgroups, and it was significantly inversed with LogHOMA-IR ( $\mathrm{r}=-0.114, \mathrm{p}=0.035)$, positively correlated with LogWBISI, LogHOMA0DI after control for age, sex, season, puberty stage $(r=0.111$, $\mathrm{p}=0.040 ; \mathrm{r}=0.122, \mathrm{p}=0.024)$. Obese patients with vitamin
D deficiency have a significantly higher risk of disturbing the glucose metabolism, such as IFG, ITG, IFG plus ITG, either IFG or ITG, for its OR 3.198(95\%CI 1.4676.97), 5.443(95\%CI 1.863-15.897), 5.560(95\%CI 1.212 25.502), 4.007(95\%CI 2.017-7.962).

\section{Conclusion}

$25(\mathrm{OH}) \mathrm{D}$ deficiencies or insufficiency are common in obese children and adolescents in Zhejiang, China. Obese patients with $25(\mathrm{OH})$ D deficiency $(<30 \mathrm{nmol} / \mathrm{L})$ are at higher risk for abnormal glucose metabolism.

\section{Authors' details}

'Department of Endocrinology, Children's Hospital of Zhejiang University School of Medicine, Hangzhou, Zhejiang, China. ${ }^{2}$ Department of Statistics, Children's Hospital of Zhejiang University School of Medicine, Hangzhou, Zhejiang, China. ${ }^{3}$ Department of Child Health Care, Children's Hospital of Zhejiang University School of Medicine, Hangzhou, Zhejiang, China.

Published: 28 April 2015

doi:10.1186/1687-9856-2015-S1-049

Cite this article as: Huang et al:: The relationship of serum

25-hydroxyvitamin D with glucose homeostasis in obese children and adolescents in Zhejiang, China. International Journal of Pediatric Endocrinology 2015 2015(Suppl 1):O49. 